\title{
Cooperación Educativa, una estrategia de relación entre la universidad y el sector productivo: la experiencia canadiense
}

\author{
Espinoza Rodríguez, Rafael L.*
}

\section{Resumen}

En el contexto internacional, se viene extendiendo la participación conjunta de las organizaciones pertenecientes al sector de la actividad económica y de las instituciones de educación superior universitarias, en la educación y entrenamiento de profesionales, con énfasis en el desarrollo de capacidades para la innovación y la aplicación de pensamiento creativo. Canadá uno de los principales paises avanzados ha encontrado en la interacción de la universidad con el sector productivo, la conformación de estrategias que promueven y concretan la participación activa de los jóvenes universitarios en procesos de aprendizaje significativo y trabajo productivo de manera integral. En este sentido en la sociedad canadiense se avanza a través de la Cooperación Educativa en la participación conjunta de la empresa, el estudiante y la universidad en la construcción del capital intelectual que fortalece su capacidad como nación para intervenir en la economia mundial. Los resultados que aquí se exponen respecto a la experiencia de la universidad canadiense en la institucionalización de los Programas de Cooperación Educativa, fueron obtenidos mediante un estudio cualitativo realizado en una muestra de universidades de ese país entre septiembre y noviembre del año 1999. Entre los datos más significativos se tienen: Canadá tiene una experiencia de mas de 30 años en el desarrollo de la Cooperación Educativa. Mas del $60 \%$ de las universidades implementan programas de cooperación educativa en diversas profesiones. La gestión de la cooperación educativa, conlleva a la creación de una infraestructura administrativa y organizacional, la misma se apoya en modelos centralizados y descentralizados. El personal responsable de conducir las funciones administrativa presenta altos niveles de formación profesional en disciplinas y habilidades para asegurar la calidad de los procesos de gestión. La implementación de programas de cooperación educativa entre algunas universidades canadienses y empresa de otros paises favorece las relaciones intemacionales. El beneficio es para las empresas, los estudiantes y las universidades genera la cooperación educativa ha sido reconocido por dependenclas del gobierno que favorece mediante programas de soporte económico la consolidación de esta estrategia de relación entre la universidad y el sector productivo.

Palabras clave: Cooperación Educativa, relación universidad sector productivo, innovación educativa, capital humano.

Recibido: 00-07-10. Aceptado: 00-11-29

* Dr. En Estudios del Desarrollo. Investigador del Doctorado en Ciencias Humanas de la Universidad del Zulia. E-mail: respinoz@iamnet.com. 


\section{Cooperative Education, a Relational Strategy Between the University and the Private Sector: The Canadian Experience}

\section{Abstract}

In the international context, there is an increasingly joint participation of the organizations that belong to the economic sector and institutions of higher education in the education and training of professionals, emphasizing the development of capacity to innovate and apply creative thinking. Canada, one of the main developed countries, has found in the interaction of the university and the productive sector, the conformation of strategies that promote and establish the active participation of university students in an integrated process of significant learning and productive work. In this manner, Canadian society advances through the Cooperative Education in the joint participation of business, students and universities in the formation of the intellectual capital that strengthens its capacity as nation to participate in the world economy. The results presented in this paper with regard to the Canadian University experience in the institutionalization of Cooperative Education Programs, were achieved through a qualitative study in a sampling of universities of this country, between September and November 1999. Among the more significant data are: Canada has more than 30 years of experience implementing Co-op Education Programs. More than the $60 \%$ of universities implement Co-op Education in different professional fields. The management of Cooperative Education is supported by an organizational and administrative structure, based on either a centralized or decentralized model. The staff responsible for managing the co-op program has higher levels of knowledge and professional skills to guarantee the quality of the inherent activities. The implementation of Co-op Education programs between some Canadian universities and business enterprises operating in foreign countries has increased the international relations. The benefits that universities, students and businesses generate in cooperative education have been recognized by governmental agencies that help with economic support programs to consolidate this strategy of relations between the university and the economic sector.

Key words: Cooperative Education, university business partnership, educational innovation, human capital.

\section{Introducción}

En el ámbito internacional el paradigma de la cooperación gana aceptación entre organizaciones que no pertenecen a un mismo sector, considerando la actividad que socialmente les corresponde ejecutar. En este sentido es notorio el acercamiento que el mundo empresarial viene teniendo hacia el mundo académico.

La empresa dirige su interés hacia la universidad por lo que ella representa como institución generadora de capital humano, para enriquecer su capital intelectual y la universidad se dispone a buscar en la empresa, un socio para conducir la formación de sus estudiantes de pregrado y postgrado con acceso a los procesos de innovación que ocurren en su seno.

La necesidad que tiene la sociedad contemporánea de disponer de creadores, innovadores, emprendedores, conlleva a que sociedades como la cana- 
diense se planteen conducir acciones que promuevan y favorezcan la creciente interacción entre el sector universitario y el sector empresarial. Entre las diversas estrategias de relación desde la dimensión de la creación de una interacción que modele la educación y entrenamiento de futuros profesionales con habilidades para fortalecer el desarrollo de la empresa canadiense establecida y generar otras nuevas, se institucionaliza la Cooperación Educativa.

En los escenarios actuales y futuros, la diversidad en las opciones que se le brindan a los jóvenes que se incorporan a las instituciones universitarias, para que éstos decidan sobre la experiencia de aprendizaje que habrán de seguir para formarse y participar inmediatamente en la sociedad como ciudadanos competentes, para producir de manera innovadora, deja de ser una decisión exclusiva de las instituciones académicas. Las expectativas y demandas del entorno crean una onda expansiva de requerimientos; para que la universidad sintonice sus funciones naturales de formación con la participación de otros, en espacios mas allá de las aulas, laboratorios y talleres universitarios.

La Cooperación Educativa como estrategia para involucrar en un ámbito dinámico a la empresa, el estudiante y la universidad, para construir la base humana, intelectual y tecnológica de países desarrollados como Canadá es el objeto de estudio que se aborda en las páginas siguientes de este artículo.

Con el apoyo del gobierno de este pais, el trabajo se condujo en el sitio, interactuando con una muestra de universidades: University of Alberta, University of
Calgary, University of Victoria y University of British Columbia. La selección de estas universidades se realizó tomando como base su exitosa experiencia en la implementación de una variedad de programas de cooperación educativa. La acción investigativa se orientó mediante la combinación de la observación y el análisis documental, con la entrevista no estructurada. La primera tarea se realizó revisando detalladamente los registros documentales emitidos por esas universidades, respecto a los modelos teóricos y operacionales que sustentan la implementación de la Cooperación Educativa. La entrevista se realizó al personal directivo de las dependencias universitarias que gestionan los programas de cooperación educativa (Decanos, Directores, Coordinadores), igualmente se entrevistó al personal de supervisión y asesoría de los estudiantes participantes en los diversos programas académicos de cooperación educativa. La información por ser básicamente cualitativa se presenta en la forma de citas textuales, presentación de figuras e ilustraciones.

\section{Naturaleza y Alcance de la Cooperación Educativa}

La sociedad contemporánea tiende a demandar de sus organizaciones la formulación de acciones que aseguren el aprovechamiento máximo de sus servicios y las haga socialmente útiles y pertinentes. Esto significa que ellas mediante el cumplimiento de una serie de procesos den lugar al logro de beneficios que trasciendan a un colectivo. En ese orden de ideas, hoy se espera que las universida- 
des y las empresas conlleven relaciones interorganizacionales; con el propósito de alcanzar beneficios mutuos $y$, algo más significativo, beneficios para la sociedad como un todo.

En el marco de estas reflexiones muchas de las universidades de los países desarrollados, entre éstos Canadá, vienen conduciendo acciones que aseguran un progresivo trabajo, compartido con las empresas en la formación y desarrollo de sus recursos humanos. Entre esas acciones se localizan estrategias novedosas como la cooperación educativa.

La cooperación educativa como estrategia institucional de las universidades, para conducir en el mundo real de trabajo, la educación y entrenamiento de los estudiantes, significa un cambio en la actitud de los miembros de estas instituciones, respecto a compartir con otros la función formadora, al igual que de la empresa al compartir sus espacios para el aprendizaje y el trabajo de los jóvenes universitarios. En el marco institucional de la relación de la universidad con el sector productivo, la cooperación educativa constituye una acción para canalizar flujos de difusión y transferencia de conocimiento desde la academia a la empresa $y$ viceversa.

Según interpretación de Gillin Murray (1993), en sentido amplio, la Cooperación Educativa es una estrategia de aprendizaje aplicado, el cual es un programa estructurado, desarrollado y supervisado por una institución académica en colaboración con una o mas organizaciones empleadoras, donde el trabajo productivo relevante es una parte integral del programa académico de los estudian- tes y es un componente esencial de la evaluación final. Tales programas pueden normalmente empezar y terminar con un período académico $y$, el componente de la experiencia de trabajo, debe involucrar trabajo productivo y comprender una proporción razonable del programa total.

La cooperación educativa es definida por Paul Fortier (1982) como una asociación de personas para un beneficio común, un proceso social dinámico en agregación ecológica (como comunidades o colonias) en el cual, los beneficios mutuos exceden en valor las desventajas de estar juntos. Según el autor, uno de los aspectos más importante de esta definición reside en el hecho de que hay muchas y diversas vías para establecer el beneficio común. En términos de la interacción que puede desarrollarse entre el sector privado y las instituciones educacionales, se debe resaltar que nunca inhibe su proceso dinámico por restricciones en el número de posibles avenidas para llevar a cabo la asociación.

En la base de la estructura de la cooperación educativa se encuentran una serie de variaciones a supuestos respecto a las cualidades y características de los socios: estudiante, empresa, universidad.

\section{El Estudiante}

Asumir la cooperación educativa, como modalidad académica para la formación en el nivel universitario, ha conllevado a una nueva y distinta valoración de la capacidad del joven adulto como responsable y capaz de ejecutar actividades 
$y$ tareas pertinentes y productivas en el medio de trabajo. En el proceso de relación de la universidad con el sector productivo a través de la cooperación educativa, el estudiante universitario representa el recurso de mayor significado y valor para posibilitar la transferencia de conocimiento desde el ámbito académico al medio productivo y viceversa.

En este sentido el estudiante que decide y opta formarse como participante en un programa de cooperación educativa debe evidenciar una serie de características personales básicas para poder asumir las exigencias y responsabilidades que implica tomar decisiones y buscar la solución de problemas, como apunta Robert Marini (1999) el joven debe poseer disposición para compartir con miembros de equipos de trabajo, tener confianza en si mismo, autonomía intelectual, disposición para desarrollar ideas.

\section{La Empresa}

En principio la empresa y sus trabajadores no están dedicados a la educación y entrenamiento de estudiantes como lo están las universidades y su personal docente. Por tanto las organizaciones que asumen la cooperación educativa para asegurar la obtención de nuevas ideas, estudiantes-trabajadores con motivaciones y disposición para innovar en la empresa han de crear las condiciones que les permita aprovechar al máximo la capacidad representada en jóvenes con habilidades para transferir conocimiento al proceso de producción de la empresa. Vale decir que se requiere la existencia de la empresa inteligente, es decir aquella que valora y consume conocimiento. Según Bruce Evans (1999), existen dos requerimientos primarios que tienen que ser contemplados para asegurar que el programa de cooperación de una organización sea exitoso. Lo primero es que ésta claramente comprenda los objetivos para participar en el programa de cooperación y que esos objetivos sean aceptados a través de la organización. EI segundo es proveer destacado apoyo y supervisión a los estudiantes del programa de cooperación.

Si bien es notorio que son las grandes empresas con visión transformadora las que usualmente consumen conocimiento, la cooperación educativa puede favorecer a la pequeña y mediana empresa que manifiesta disposición para innovar en sus procesos cuando esta acuerda con la universidad la creación de condiciones básicas para que los estudiantes apliquen sus conocimientos y validen en lugar de trabajo la teoría.

Según la información aportada por varios miembros ${ }^{1}$ de las dependencias universitarias que gestionan la cooperación educativa, las empresas que manifiestan mayor disposición para demandar estudiantes son aquellas que están dispuestas a suscribir con los jóvenes y la universidad el compromiso de colocarlos en puestos de trabajo donde se incrementa los niveles de responsabilidad y de utilización de conocimiento.

1 Thomas, Johan, University of Victoria, Janice Attersiey, University of Calgary, Cludette J. De Bruijin, University of Alberta, 1999. 


\section{La Universidad}

La Cooperación Educativa introduce en la universidad diversos tipos de exigencias. Una de primer orden, la representa mayor apertura hacia el concepto de educación y entrenamiento de los estudiantes. La formación en el nivel universitario en estrecha relación con el medio laboral expande las posibilidades de aprendizaje significativo ligado a la cotidianidad de la organización del trabajo productivo. El desarrollo de habilidades prácticas ligadas al conocimiento teórico es requerimiento de la vida contemporánea, la exigencia que se le hace a la educación universitaria es que evidencie su alcance en la acción, en el hacer.

\section{Valores Educacionales, Sociales y Económicos de la Cooperación Educativa}

La emergencia de una sociedad que está asumiendo el cambio, la transformación como una condición permanente de su quehacer económico, científico, social, político y particularmente educativo, ha dado lugar a un replanteamiento respecto a la independencia $y$ complementariedad entre las instituciones que la conforman. En ese sentido ocupa un lugar el creciente interés respecto a la interacción de la universidad y la empresa a través de la formación de los profesionales.

En el ámbito de la sociedad del conocimiento, en la que actualmente vivimos, la empresa y la universidad conducen procesos que generan requerimientos en doble dirección. La empresa inte- ligente para conformar su capital intelectual depende de la disponibilidad de trabajadores del conocimiento que le soporten en la innovación que le es necesaria, así mismo la institución académica necesita de medios apropiados para validar la pertinencia y utilidad del conocimiento y las habilidades transferidas al estudiante así como también acceso a la información y tecnología con la que se trabaja en el medio productivo para abordarla como parte del aprendizaje del estudiante. En este marco de interdependencia se viene imponiendo la búsqueda de las alianzas interorganizacionales -Universidad \& Empresa-.

El clima actual en la educación superior se ha centrado en la formulación de políticas que orienten y hagan factible la participación activa y dinámica de los académicos y estudiantes en la demostración temprana y permanente de los resultados del aprendizaje logrado en el salón de clase y los laboratorios, en la consecución de nuevos espacios, de recursos materiales y personal de alto nivel profesional en el medio externo para compartir la compleja tarea de educar y desarrollar habilidades para el aprendizaje permanente, la toma de decisiones, la aplicación productiva del conocimiento y habilidades para la innovación. Esta preocupación hoy se localiza en paises industrializados compitiendo en la economía globalizada y está representada en una propuesta que ha sido altamente exitosa como lo es la cooperación educativa, a la cual se le reconoce la generación de una serie de valores para la sociedad. 


\section{Valores Educativos}

La capacidad de los paises industrializados para avanzar hacia niveles de desarrollo pertinentes y en sintonía con características como la transitoriedad y la complejidad que están presentes en casi todos los procesos que en ellas se llevan a cabo suponen que tanto los individuos como las organizaciones desarrollen habilidades y actitudes para el aprendizaje significativo, permanente. En este sentido vale decir, que hay un cambio en la concepción de la actividad educativa, de los actores involucrados, de los medios, recursos y espacios donde se conducen los procesos de aprendizaje. Como expresión de cooperación educativa resalta el reconocimiento que cualquier organización tiene un conjunto de conocimientos y habilidades técnicas que puede compartir con la universidad para la formación de los futuros profesionales.

\section{Valores Sociales}

En el devenir del siglo XXI la sociedad de los países desarrollados buscan construir mecanismos que fortalezcan su capacidad para generar servicios y bienes que agreguen valor a las condiciones de vida de sus ciudadanos. En este sentido se viene avanzando en una redefinición del rol que deben cumplir sus instituciones. El énfasis en compartir el compromiso de sustentar procesos de valor e impacto nacional, tales como desarrollar la infraestructura humana que conciba, modele y ejecute el conocimiento y la tecnología, demanda el desarrollo de valores sociales como: la solidaridad, la disposición a fortalecer la capacidad del individuo y las instituciones y la corresponsabilidad.
La educación cooperativa con sus principios teóricos conileva la asimilación y práctica de tales valores. La acción cooperativa conduce a que las instituciones y personas involucradas actúen con base en tales principios dando lugar a acciones identificables como expresión de los valores.

La solidaridad entre las organizaciones y personas pueden lograrse cuando existe identificación con propósitos y objetivos de beneficio para todos. A través de la cooperación educativa se promueve la solidaridad para apoyar y construir la fortaleza de un país para desarrollar la actividad económica productiva, así como también el desarrollo de la innovación para producir.

La disposición de las instituciones para fortalecer la capacidad de individuos $y$ otras instituciones para construir las condiciones que conllevan a lograr el bienestar de todos los ciudadanos, es uno de los productos sociales que se puede asociar a la implementación de la cooperación educativa.

La corresponsabilidad de la empresa y la universidad para garantizarle al pais la dotación de ciudadanos educados, entrenados para la actividad productiva, es uno de los valores sociales que mayor trascendencia tiene para la cohesión de los dos tipos de organizaciones que construyen la base para la construcción del capital humano e intelectual de la sociedad.

\section{Valores Económicos}

El crecimiento y solidez de la actividad económica de los países está dependiendo de manera significativa de la ca- 
pacidad de sus trabajadores para introducir innovaciones particulares. La innovación como cualidad humana que debe ser desarrollada con el propósito de satisfacer necesidades o mejorar procesos, productos, servicios, tiende a resaltarse como uno de los aspectos de alto valor económico para la sociedad contemporánea. Otra cualidad humana, que la dinámica de la actividad económica reclama, es la de emprendedor. Las expectativas sobre el incremento de miembros de la sociedad con conocimiento y habilidades para contribuir en la apertura de sus propios negocios se instala de tal manera, que la cooperación educativa propicia la formación de futuros innovadores y emprendedores.

Otro aspecto del alcance de la cooperación educativa como aporte al desarrollo de valores económicos, es el fortalecimiento de actitudes como la responsabilidad por el trabajo productivo y la eficiencia, entre otras.

\section{EI Programa de Cooperación Educativa Canadiense en el contexto del Sistema Nacional de Formación en el nivel universitario}

El inicio de la educación cooperativa en Canadá se sucede en el año 1957. La University of Waterloo en Ontario fue la primera institución en establecer los programas de Cooperación Educativa. A partir de ese momento la cooperación educativa ocupa un lugar relevante entre las estrategias de formación profesional tanto en el pregrado como en el postgrado.
El reconocimiento de la pertinencia que para la sociedad canadiense tiene la cooperación educativa se puede estimar si se considera el número de instituciones de educación superior que han incorporado programas de cooperación educativa. Según dato suministrado por la Canadian Association for Co-operative Education hasta principios del 2000 en esta organización participan como miembros 50 universidades (CAFCE, 2000) de un total aproximado de 68 universidades existentes en ese país.

La relevancia y contribución que la cooperación educativa ha tenido para las instituciones involucradas en la institucionalización de esta estrategia se recoge a partir de la reseña de apreciaciones y reflexiones:

\section{A. Sector Empresarial}

- El Programa de Cooperación Educativa de Negocios de la University of Alberta es una excelente asociación entre la Universidad, sus estudiantes y empleadores. Nosotros empleamos ocho meses, estudiantes para nuestro departamento de contabilidad. Esto nos proveyó con una oportunidad para alcanzar no solamente el trabajo diario y proyectos completos, sino también para evaluar los estudiantes para posibles empleos futuros. Los otros aspectos del programa permiten la discusión entre la universidad y la industria acerca de habilidades requeridas para satisfacer trabajos actuales y futuros. Nosotros hemos estado y continuaremos siendo positivos soportes de ese programa" (Treacy Maurine. Human Re- 
source Representative. Syncrude Canada Inc.)

- "GE Capital Canada ha sido un soporte del Programa de Cooperación Educativa de la Facultad de Negocios desde su inicio. Nosotros hemos encontrado siempre nuestros estudiantes de cooperación dispuestos a contribuir de manera muy rápida con nuevas ideas, habilidades técnicas, entusiasmo y energía para nuestro lugar de trabajo. La experiencia de cooperación ha posibilitado a nuestra organización desarrollar y evaluar los potenciales futuros gerentes a la manera de análisis costo-beneficio. Reclutar a través de la Oficina de Servicios de Colocación siempre ha sido un gusto, debido en gran parte por la extrema ayuda, del conocedor y eficiente staff" (Kelly Blackett - Human Resource Specialist GE Capital Ltd.)

- "El Centro de Cooperación Educativa de Ingeniería de la University of Alberta provee excelentes servicios. Yo he estado empleando de uno a tres estudiantes cada periodo de trabajo en los últimos 10 años. El staff de Cooperación Educativa entiende que ellos tienen dos clientes - estudiantes e industrias. Ellos hacen un gran trabajo de unirlos antes a ambos" (Mike Kardash Peng. Celanese Canada Inc. Edmonton).

\section{B. Estudiantes y Egresados}

Desde la perspectiva de los estudiantes y egresados la cooperación educativa les ha reportado beneficios. Esto se evidencia de una serie de testimonios de jóvenes de la University of Calgary:
- "El programa de cooperación educativa me ha dado la oportunidad para sentirme seguro acerca de mi decisión educativa. Esta experiencia me proveerá también con una ventaja competitiva, no solo en el mercado de trabajo después de la graduación, sino en el salón de clase para el resto de mis cursos." (Chris Bartole. Estudiante de Gerencia Petrolera. Talisman Energy Inc.)

- "El conocimiento que he ganado en The Canadian Space Agency en mi primer periodo de trabajo es irremplazable. Desde extender la mano al primer Canadiense en volar al espacio hasta ser una parte del equipo que hace que todo ello sea posible - la experiencia ha sido increible". (Clarissa Von Stetten. Estudiante de Negocios Internacionales. The Canadian Space Agency)

- "A través de mi período de trabajo yo he aprendido habilidades que nunca hubiese obtenido en el salón de clase. Este ha sido un gran lugar para mí, para empezar mi carrera". (Jennifer Yee. Canadian Pacific Railway)

Los argumentos en reconocimiento a la pertinencia de formación profesional a través de la cooperación educativa tienen distintos matices. Esto se recoge de la valoración de estudiantes de la University of British Columbia.

- "A través del Co-op Japan program, yo he asegurado un empleo con Kawasaki Steel Corp. (Kawatetsu) de Japón para mis períodos de trabajo de verano y otoño. Yo he estado trabajando en Kawatetsu hasta ahora por 2 meses, y me he adaptado a mu- 
chos de los aspectos de la cultura Japonesa y su medio ambiente de trabajo... mi trabajo es investigar y mejorar la tasa de enfriamiento del acero que procede de los hornos... (Perry Wong. Programa de Ingeniería Mecánica).

- "Finalizado mi último año del programa de cooperación educativa de Ingeniería Eléctrica y Computación en la UBC, agradezco por las oportunidades que me han ofrecido. Además del conocimiento en los libros y la participación extracurricular, yo me he aventurado en una expedición de por vida para explorar e investigar diferentes culturas. A través del Programa de Cooperación Educativa de Ingeniería y otros programas conjuntos, conduje períodos de trabajo en Hong Kong, Japón, Alemania, Vancouver y el Valle del Silicón de Canadá" (Winnie Cheng, Ingeniero Eléctrico)

\section{Académicos}

El respaldo brindado por los académicos a la implementación de los programas de cooperación educativa, refuerza la valoración que la universidad tiene de la pertinencia social de esta estrategia universitaria para la formación de los profesionales. La actitud de los universitarios se evidencia a través de las siguientes reseñas:

- "... hay solamente dos razones por las cuales nuestros estudiantes son tan bien preparados para el mercado de trabajo. Los laboratorios, equipados con siete diferentes estaciones experimentales que son todas construidas con modernos controles $y$ sensores industriales, proveen a los estudiantes con oportunidades para obtener experiencia y su habilidad para traer reales ejemplos industriales al salón de clase, todo combinado para crear una educación que trabaja" (Dr. Fraser Forbes, profesor del programa de Control de Procesos Computarizados - University of Alberta)

- “... Es una innovación, que involucra a la institución para satisfacer una fuerte necesidad de percepción nacional... Es un ejemplo de cómo nuestros graduados tomarán mejores ventajas del abastecimiento de las maderas de Canadá y crea nuevas oportunidades en el mercado de trabajo global" (Dr. John McLean - Decano de la Facultad de Forestales University of British Columbia)

A manera de conclusión preliminar se puede señalar, que desde la perspectiva nacional la implementación del programa de cooperación educativa conlleva a diseñar, implementar y evaluar una infraestructura soportada por los principios sustantivos del aprendizaje, la transferencia de habilidades, la aplicación del conocimiento, la generación de resultados, corresponsabilidad en el cumplimiento de los compromisos materiales y no materiales.

\section{La Organización y Gestión de la Cooperación Educativa}

El diagnóstico revela que es variable el crecimiento de la adopción de la cooperación educativa entre las universidades, asi se tiene que mientras algunas lo 
tienen extendido a todas las Facultades como única alternativa de formación profesional (University of Waterioo), otras lo ofrecen en todas sus Facultades pero como alternativa frente al modelo de formación usual. En otros casos, sólo algunas Facultades ofrecen programas de cooperación educativa. El crecimiento institucional de la cooperación educativa también se observa en el carácter obligatorio u opcional con que lo ofrece la universidad. Asi se tiene que en algunas universidades ciertas carreras se implementan solamente por cooperación educativa, siendo obligatorio para aquellos estudiantes que quieren realizar esa carrera en esa universidad hacerlo bajo esta modalidad (Facultad de Ingeniería de la University of Victoria), La forma más extendida en la mayoría de las universidades es presentar la cooperación educativa como alternativa para que los estudiantes asuman la opción en base a sus necesidades, intereses, expectativas.

La mayor o menor extensión de la cooperación educativa en cierta medida delinea el modelo de organización y gestión asumido. Asi se tienen dos grandes variantes, el centralizado y el descentralizado.

En el modelo centralizado la estructura la define la creación de una dependencia con denominación de instituto o centro para la cooperación educativa, localizado al mismo nivel de las Facultades o Escuelas. La misma reporta al nivel de vice-presidencia académica de la universidad. Para conducir esta dependencia se dispone del cargo de director. En este modelo las Facultades que ofrecen los programas de cooperación educativa, siguen lineamientos del nivel superior el cual lo integra un consejo asesor.

Las Facultades cuentan con la figura del gerente y/o coordinador de cooperación educativa y cada programa en particular dispone de coordinadores. El centro de cooperación educativa coordina con las Facultades a través de sus coordinadores de cooperación educativa la gestión hacia el sector externo (Diagrama No. 1).

En el caso de las universidades donde solo algunas Facultades disponen de programas de cooperación educativa, el nivel de descentralización es mayor. Cada Facultad es autónoma en la organización, y gestión de los programas de cooperación educativa en este caso se cuenta con el cargo de Decano de cooperación educativa y este reporta al Decano de la Facultad. Cuando la Facultad ofrece varias carreras a través de cooperación educativa se dispone de coordinadores para cada programa. (Diagrama No. 2 y No. 3).

En la gestión de los programas de cooperación educativa la conformación del equipo humano que intermedia en el proceso de formación del estudiante es variable dependiendo del nivel de descentralización del programa. (llustración No. 1)

El proceso de gestión de la cooperación educativa, descansa en la disposición y aplicación de una serie de instrumentos normativos, tales como un cuerpo de politicas que orientan el desenvolvimiento de todos las instituciones, dependencias e individuos participantes: docentes, estudiantes, personal administrativo, coordinadores, supervisores, consejeros.

De igual manera se requiere la utilización de una variedad de medios y re- 

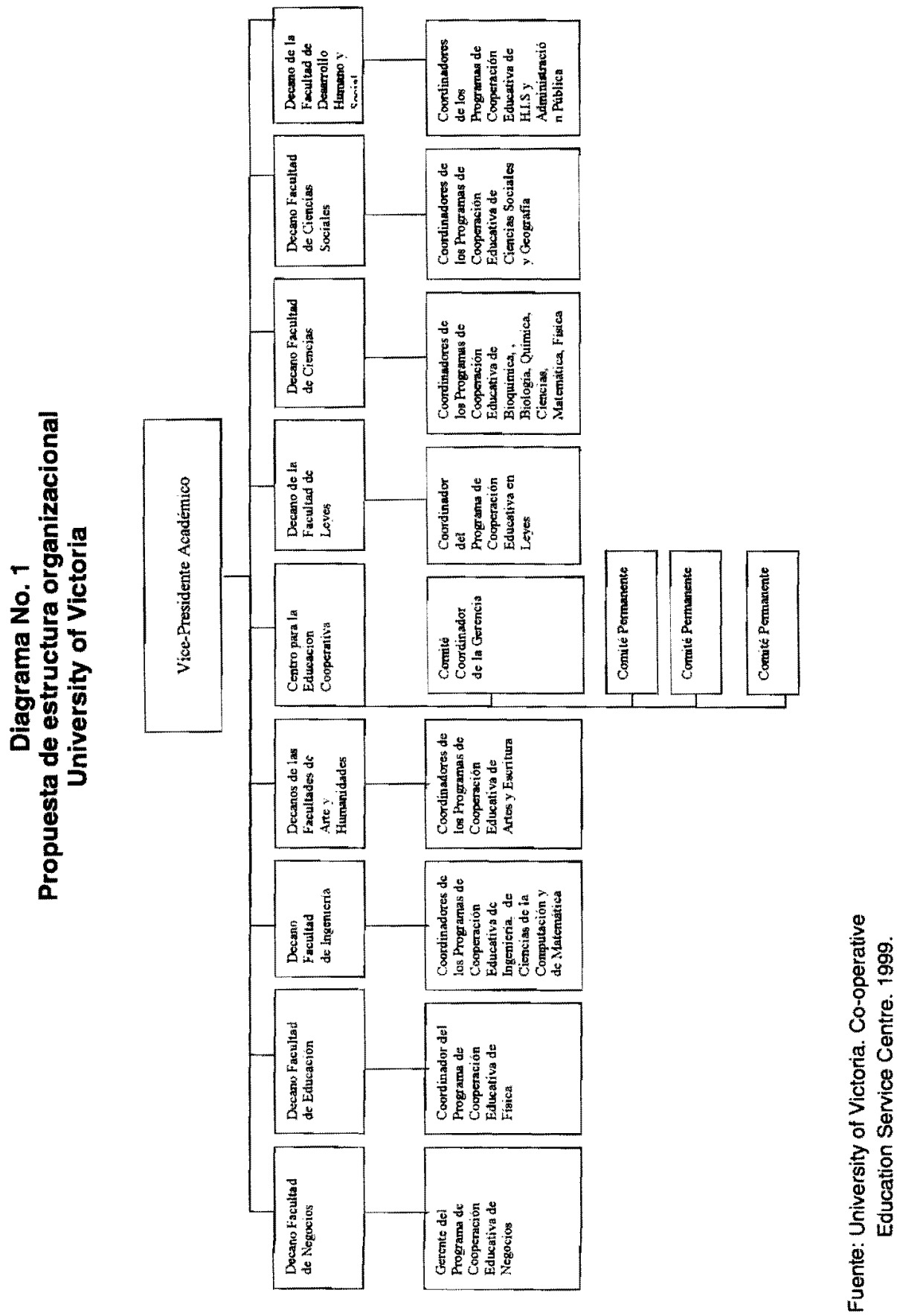

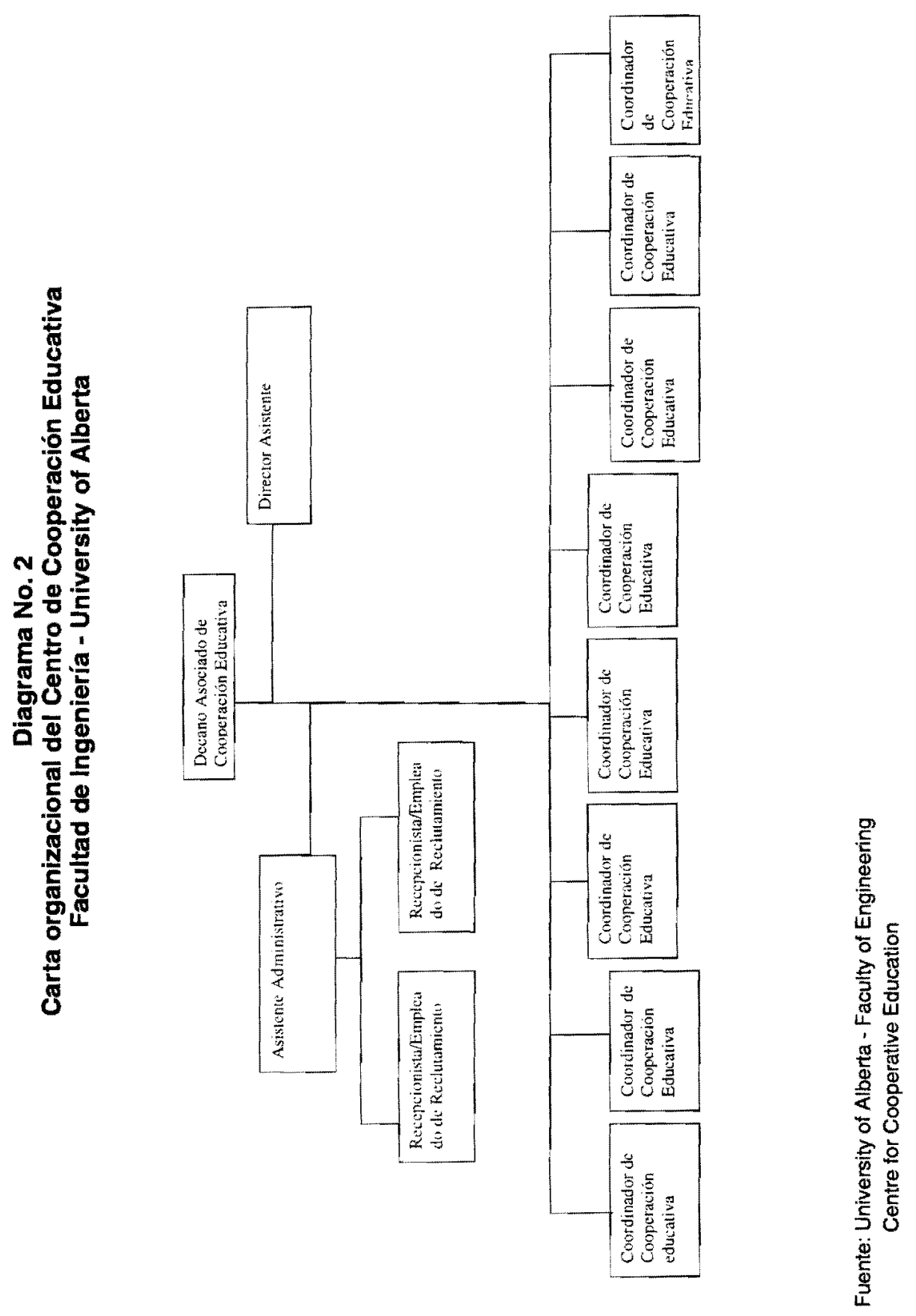


\section{Diagrama No. 3}

\section{University of Calgary - Management Career Centre Organigrama organizacional}

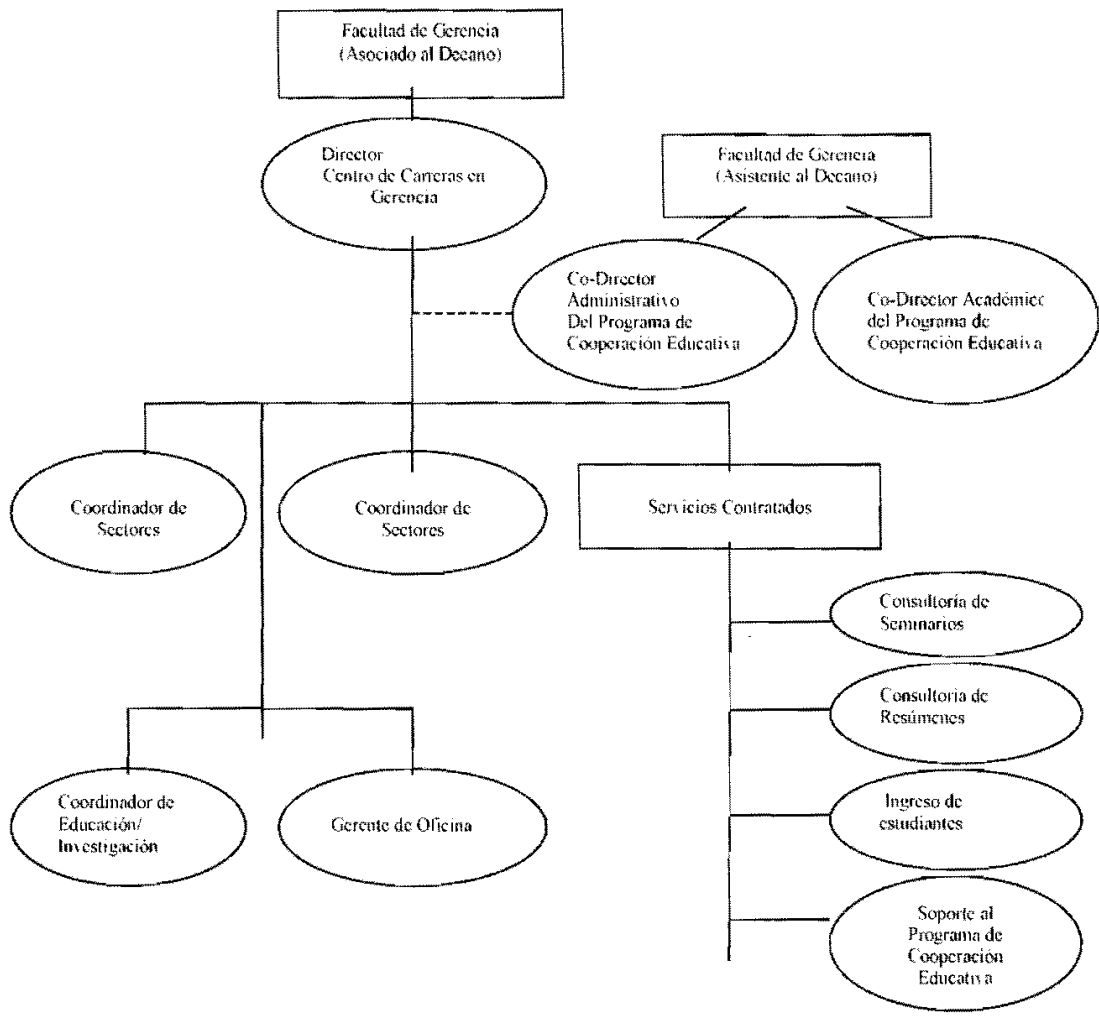

Fuente: University of Calgary - Career Service. 


\section{llustración No.1 \\ Equipo de soporte del Estudiante de Cooperación Educativa}

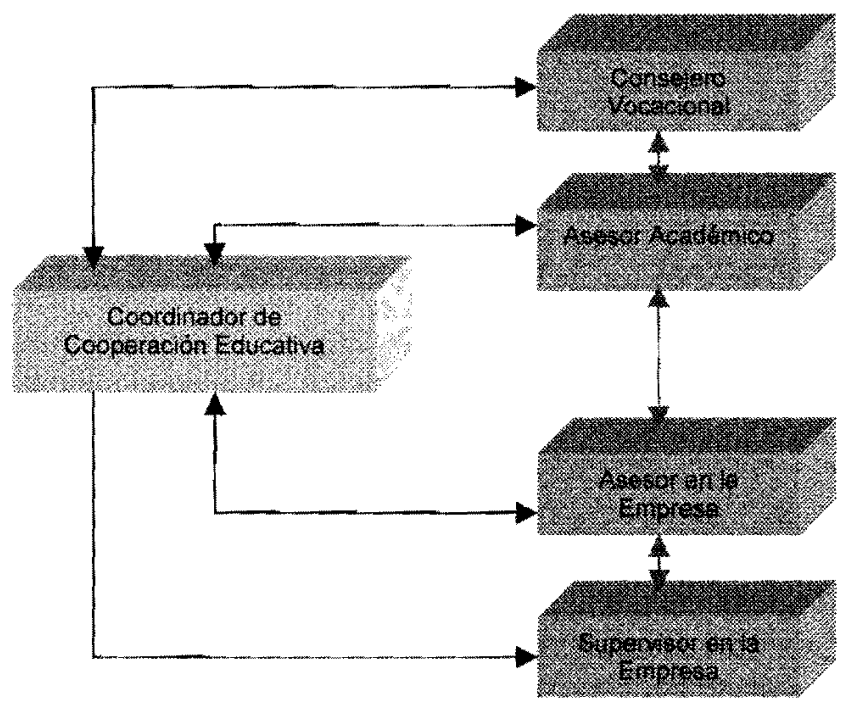

Fuente: Elaboración del Autor.

gistros administrativos para asegurar la eficiencia de los procesos correspondientes a la interacción con los estudiantes del programa y las empresas participantes. En el desarrollo del programa de cooperación educativa se pueden distinguir tres grandes fases por las que transcurre el estudiante, el inicio o fase de inducción, la fase de preparación para el inicio del primer período de trabajo y la fase de integración a la actividad de aula posterior a cada experiencia de trabajo. Para asegurar el desarrollo gradual y progresivo del estudiante las universidades han diseñado una serie de medios y recursos que se puede clasificar en recursos para proveer información institucional al estudiante, recursos para desarrollar habilidades para el desempeño en el medio laboral y recursos para evaluar el desempeño en el medio laboral. Al respecto se cuenta con los siguientes instrumentos:

- Manual para el estudiante

- Manual para el empleador

- Reporte de evaluación del empleador

- Reporte de evaluación del docente asesor

- Formas de asignación de empleo

- Reporte de visitas al sitio de empleo

- Cronograma del programa

- Hoja de información del estudiante del período de trabajo

- Reporte técnico de suspensión del estudiante del programa

- Normas para la elaboración de reportes técnicos 
- Informe de evaluación de la Oficina del Decano sobre el período de trabajo de los estudiantes

- Informe de evaluación de la Oficina de Cooperación Educativa

- Sobre el período de trabajo de los estudiantes

- Regulación de acuerdos confidenciales sobre propiedad intelectual y reserva de información por el estudiante

Los datos presentados, revelan que cada universidad conduce procesos de gestión que garantiza la calidad y eficiencia de sus programas, de igual manera, toma en cuenta no sólo las expectativas $y$ necesidades de los estudiantes, sino muy particularmente las del sector empleador en tanto que es un aliado en el desarrollo de la calidad de la educación de ese país, y la existencia de un mercado académico cada vez más competitivo.

Un aprendizaje que nos deja el diagnóstico de la organización y gestión de la cooperación educativa, es asimilar la conformación de un sistema que incorpora las nuevas tecnologías de la comunicación y la información como soporte de tan minuciosa y compleja tarea. Ese sistema-red coloca a los tres usuarios de la cooperación educativa en una situación de permanente interacción, para la búsqueda de oportunidades de empleo y colocación de estudiantes en las instituciones empleadoras, para oferta de puestos de trabajo por los empleadores, al igual que para el soporte y respaldo que la universidad debe brindar al estudiante hacia los lugares de trabajo donde este se encuentre y a las empresas necesitadas de conocimientos particulares para desarrollar sus actividades productivas. (llustración $\mathrm{N}^{\circ}$ 2)

\section{La Certificación Nacional del Programa de Cooperación Educativa}

La institucionalización de la cooperación educativa y su desarrollo en Canadá es la consecuencia del modelo asumido por las instituciones educacionales y las de soporte para asegurar la pertinencia y calidad.

La mayoría de las universidades canadienses que implementan programas de cooperación educativa valorando la importancia que para ellas tiene asegurar la calidad de los mismos, como respaldo al compromiso de ofrecer a la sociedad servicios educativos de alto nivel, han acogido la certificación de que son objeto sus programas por un agente externo.

Desde su inicio en 1973, la Asociación Canadiense para la Educación Cooperativa (Canadian Association for Co-operative Education, CAFCE), ha sido un defensor $y$ asesor de académi$\cos$, estudiantes, y empleadores involucrados en el proceso de cooperación educativa a través del país.

En 1979 la CAFCE estableció un procesos para la acreditación de los programas de cooperación educativa. El proceso de acreditación en si mismo es importante en tanto que establece criterios que son aceptados como niveles de exigencia para los programas y su gestión, de igual manera para mantener 0 adecuarse a aquellos criterios y necesidades de la sociedad, empleadores y los estudiantes participantes (CAFCE, 1996). 


\section{Ilustración No. 2 \\ Red de interacción estudiante - universidad - empresa}

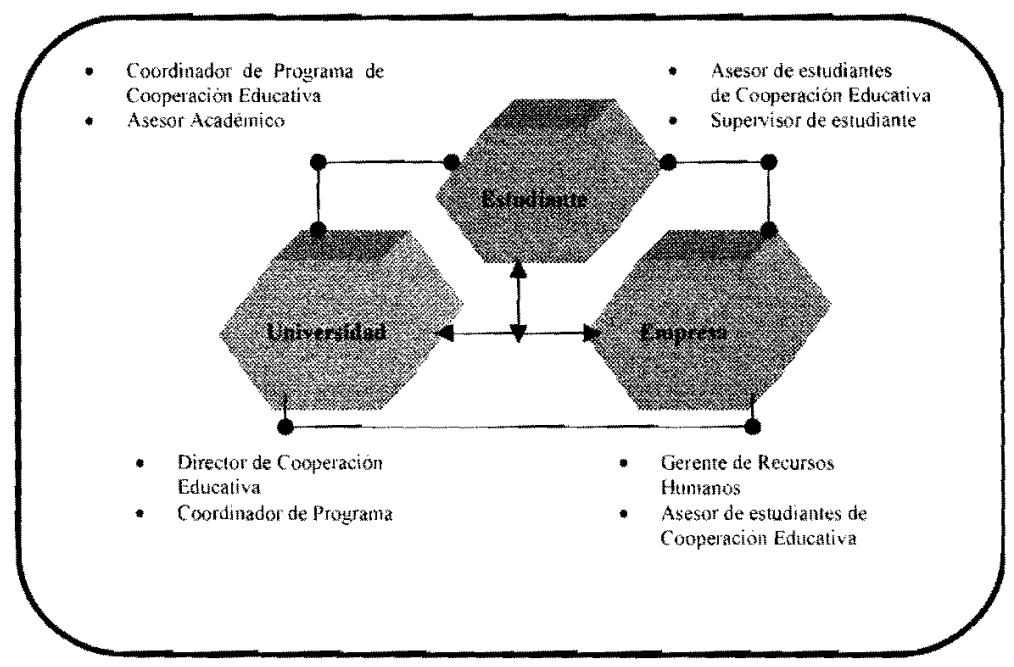

Fuente: Elaboración del Autor.

El propósito del proceso de acreditación es demostrar que el programa que solicita la acreditación reúne los criterios y niveles que son requeridos para asegurar la calidad del programa de cooperación educativa.

En asociación con la CAFCE, profesionales de las universidades, otras instituciones de educación superior y el sector de negocios activamente promocionan la comprensión y beneficios de la educación cooperativa. La CAFCE es proactiva en el avance de la posición de las instituciones educativas, el gobierno y los empleadores interactuando en la cooperación educativa.

\section{La Cooperación Educativa soporte de las relaciones Internacionales Canadienses}

En el ámbito de las relaciones internacionales, las instituciones de educación superior canadienses desempeñan una función de primer orden. Las universidades conscientes del indetenibie proceso de globalización económico, científico, cultural, educacional y laboral que se impone en el planeta, tienden a reforzar las estrategias concebidas a nivel gubernamental con el propósito de asegurar la participación competitiva de Canadá en los escenarios internacionales. 
El gobierno de Canadá ha creado los mecanismos para que las universidades que disponen de programas de cooperación educativa puedan gestionar en el ámbito internacional la consecución de espacios para que los estudiantes tanto de pregrado como de postgrado accedan a oportunidades de aprendizaje y trabajo productivo en sociedades y culturas diferentes. En este sentido en Mayo de 1991, con respaldo del gobierno canadiense fue establecido un convenio entre este país y Japón para un intercambio mutuo de largo plazo respecto a oportunidades científicas e industriales. Un consorcio de cuatro centros de universidades canadienses fue formado para implementar el programa, iniciado en una fase piloto de tres años.(CAFCE, 1999) El denominado Co-op Japan Program es un programa nacional de las universidades canadienses, el programa de colocación internacional integra algunos de los mejores estudiantes, investigadores, y profesionales que Canadá puede ofrecer, con altos comisionados de negocios e industrias participantes de los dos países.

Para el momento de finalización de la fase piloto del convenio, 175 colocaciones habian sido completadas con estudiantes y compañías, ambos reportando que sus expectativas habian sido superadas (CAFCE, 1999).

En 1994, con el éxito de la fase piloto sustentada, el Gobierno de Canadá produjo un mandato para la expansión. Cada universidad fue invitada a participar, convirtiendo el Co-op Japan Program en un real programa nacional de colocación. Actualmente 22 universidades participan y el número se incrementa.
Un promedio de 60 estudiantes participan cada año, dedicando más de 11 meses trabajando en investigación básica y desarrollo orientado del medio ambiente de mas de 50 compañias del Japón (CAFCE, 1999).

El esfuerzo de la universidad canadiense para brindarle al estudiante, a través de la cooperación educativa, oportunidades para que desarrolle una visión y experiencia internacional ha dado lugar a que los coordinadores de los programas desarrollen acciones para lograr una expansión de los países dispuestos a abrir sus fronteras para la colocación de estudiantes en empresas de esos países. Algunas referencias al respecto son las siguientes:

En el último año, cerca de 2.500 estudiantes de 35 departamentos de la University of Victoria trabajaron dentro de 120 diferentes instituciones privadas y de gobierno en 28 diferentes países (Reeve, 1999).

Al respecto la University of Victoria registra algunas experiencias: "El programa de Cooperación Educativa en Geografía ha forjado nuevos vínculos de investigación en Rusia. Un proyecto de investigación conjunta fundado por la Fundación Gorbachev estuvo basado en un período de cooperación iniciado a partir de 1991, cuando June Whitmore, Coordinadora del programa de Geografía, inició un acuerdo de intercambio de estudiantes entre la Moscow State University y la University of Victoria. Dos estudiantes de la Moscow State University intercambiaron con la University of Victoria en 1991 y 1994. En 1992, las actividades fueron ampliadas para incluir al Jefe del 
Departamento de Biogeografía, Dmitri Krivolutsky, quien visitó la UVic por una semana. ... Dos estudiantes canadienses de cooperación educativa tuvieron la oportunidad de viajar y trabajar en Rusia en 1997" (Whitmore, 1999).

\section{La trayectoria de algunas universidades en el fortalecimiento de la Cooperación Educativa en Canada}

La fortaleza de las universidades en la implementación de la cooperación educativa en Canadá se puede apreciar a partir del crecimiento alcanzado en la muestra que se reseña.

\section{University of Victoria}

En la University of Victoria, el programa de cooperación educativa tiene sus inicios a partir de 1975, año en que fue aprobada su introducción, e iniciado formalmente en al año 1976.

La University of Victoria destaca por su fortaleza en la planificación y gestión de una estructura flexible y dinámica. Su programa de cooperación educativa es el tercero más grande en Canadá. EI modelo de cooperación educativa es ofrecido en todas las Facultades, generando estudiantes de más de 40 Departamentos y con más del $30 \%$ de su población de estudiantes a tiempo completo participando en educación cooperativa. En el año académico 1997-1998, los estudiantes de cooperación educativa participaron en 2.936 períodos de trabajo con más de 1.000 empleadores; desde 1976 el programa de cooperación educativa ha gestionado un total acumulado de 24.677 colocaciones de periodos de trabajo. El número de estudiantes colocados en 20 años fue de 18.940. (UVIC Co-Operative Education Programs $20^{\text {th }}$ Anniversary)

Actualmente viene formando profesionales a través de los programas de cooperación educativa: que se exponen en la Tabla 1.

\section{University of Calgary}

La University of Calgary da inicio a la cooperación educativa a partir del año 1989 con 60 estudiantes en seis programas. Desde ese momento el crecimiento ha sido acelerado tanto en programas como en el número de estudiantes, en ese sentido hasta febrero de 1999 ofrece los siguientes programas de Cooperación educativa donde participan 600 estudiantes:

Química Aplicada, Física Aplicada, Ciencia Actuarial, Estudios de la Comunicación, Estadistica, Estudios Religiosos, Francés, Inglés, Filosofía, Estudios del Desarrollo, Historia, Sociología, Ciencia Política, Lingũistica, Geografía, Economía, Geologia, Geofísica, Geología Ambiental Aplicada, Ecología, Gerencia General, Recursos Humanos y Dinámica Organizacional, Negocios Internacionales, Sistemas de Gerencia de Información, Mercadeo, Gerencia de Operaciones, Gerencia Petrolera, Gerencia de Aiesgos, Seguros y Beneficios del Trabajador, Gerencia de Turismo y Hospedaje, Gerencia de Turismo y Hospedaje y Mercadeo, Finanzas, Contabilidad. 


\section{Tabla 1 \\ University of Victoria \\ Oportunidades de Estudios}

Facultad Ingeniería

- Computación

- Eléctrica

- Mecánica

- Ciencias de la

Computación

\section{Facultad Arte}

- Historia del Arte

- Música

- Teatro

- Artes Visuales

\section{Facultad Leyes}

- Leyes

\section{Facultad Negocios}

- Comercio

Desarrollo Humano y Social

- Información de Ciencias de la salud

Facultad Ciencias

- Bioquimica/Microbiología

- Biología

- Química

- Ciencias de la Tierra y Ciencias del Océano

- Matemática

- Fisica

\section{Facultad Educación}

- Kineseología

- Administración de Servicios Recreativos

\section{Facultad Humanidades}

- Inglés

- Francés y Literatura

- Estudios Germánicos

- Estudios Griegos y Romanos

- Estudios Hispánicos e Italiano

- Historia

- Historia del Arte

- Lingüística

- Estudios Medievales

- Estudios del Pacífico y Asia

- Filosolía

- Escritura Profesional

- Estudios Eslavos

- Estudios de la Mujer

- Escritura

\section{Estudios para Graduados}

- Economía Aplicada

- Administración de Negocios

- Estudios de Gerencia

- Administración Pública

\section{Facultad Ciencias Sociales}

- Antropología
A. Economia
B. Estudios del Medio Ambiente
C. Geografía
D. Ciencias Políticas
E. Psicología
F. Sociología

Fuente: Tripticos de la University of Victoria. Elaboración propia.

Un indicador del progreso de algunos de sus programas es la cantidad de estudiantes que son colocados anualmente en el sector empleador. Así se tiene el caso del programa de Gerencia, el cual coloca mas de 200 jóvenes en empresas tanto de Calgary como de otras re- giones del país (University of Calgary, Management News-letter, 1999).

\section{University of Alberta}

La educación cooperativa en la University of Alberta tiene sus inicios en el año 1981 con la implementación del pro- 
grama en ingenieria que es el segundo más grande en el país. La demanda de los estudiantes para cursar los programas del área de ingeniería por cooperación educativa se traduce en cifras significativas, aproximadamente el $50 \%$ de los estudiantes de la Facultad de Ingeniería se encuentran registrados en los programas de cooperación educativa. Otro dato relevante es la cantidad de estudiantes que coloca en puestos de trabajo, cerca de 1000 experiencias de trabajo se gestionan anualmente a través de la Provincia de Alberta y el resto de Canadá (Faculty of Business, Office of Placement Services, 1998).

La Facultad de Negocios es la otra dependencia que administra programas de cooperación educativa. Desde el año 1991 y con crecimiento continuo se desarrolla la diversificación de las opciones que gestiona esta Facultad. En los nueve años de actividad cerca de 200 organizaciones empleadoras han participado empleando jóvenes de los programas (Cooperative Education Newsletter, 1991). Que se expone en la Tabla 2.

\section{University of British Columbia}

En el año 1978 la Universirty of British Columbia da inicio a la cooperación educativa. En 21 años de trayectoria la experiencia acumulada por esta universidad la coloca entre las que han desarrollado una amplia gama de programas en diversos campos de la formación profesional. El programa más reciente es el de Ciencias en el Procesamiento de la Madera, implementado por la Facultad de Forestales, en mayo de 1999 egresaron los primeros 10 profesionales, que se exponen en la Tabla 3.

\section{Consideraciones Finales}

El estudio de la experiencia canadiense en la institucionalización de la Cooperación Educativa, nos permite llegar a las conclusiones siguientes: En primer lugar se registra una definitiva confianza por parte de la empresa en la calidad intelectual y cualidades personales de los jóvenes estudiantes universitarios, como personas capaces de agregar valor con su trabajo e ideas a sus negocios. En segundo lugar se hace evidente la vocación de la universidad para interactuar con la empresa en el proceso de formación de los profesionales requeridos por la economía de ese país, sumando, la participación del sector empresarial en la revisión del corpus de conocimientos y habilidades intelectuales que deben transferirse mediante la función educativa a los estudiantes. En tercer lugar es necesario destacar que la expansión de la cooperación educativa entre las universidades canadienses refleja el grado de madurez alcanzado en la relación entre la universidad y la empresa a propósito de consolidar procesos que fortalezcan las organizaciones productoras y académicas como soporte para el desarrollo social.

La cooperación educativa, como modelo de formación universitaria se puede valorar pertinente para aplicarlo a la realidad universitaria venezolana. En el futuro inmediato, las universidades $y$ las empresas nacionales tenderán a necesitarse unas de otras, en aspectos tales como la disponibilidad de espacios para 


\section{Tabla 2 \\ University of Alberta Oportunidades de Estudio}

\begin{tabular}{|c|c|}
\hline Facultad de Ingenieria & Facultad de Negocios \\
\hline $\begin{array}{ll}\text { - } & \text { Química } \\
\text { - } & \text { Coivil } \\
\text { - Eléctrica } \\
\text { - Mecánica } \\
\text { - Materiales } \\
\text { - Minas } \\
\text { - Petróleo }\end{array}$ & 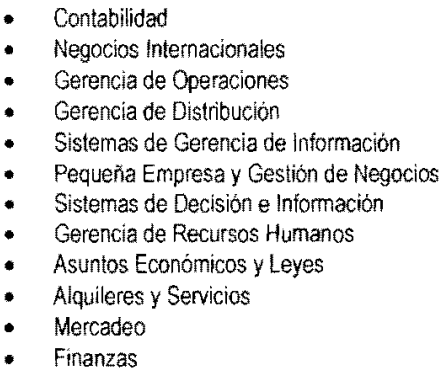 \\
\hline
\end{tabular}

Fuente: Tripticos de la University of Alberta. Elaboración propia.

\section{Tabla 3}

\section{University of Victoria. Oportunidad de Estudio}

\section{Facultad Ingenieria}

- Química

- Civil

- Computación

- Eléctrica

- Geológica

- Mecánica

- Electro-Mecánica

- Física

- De los Metales

y Materiales

- De Minas y Procesos

Minerales

- Ambiental

\section{Facultad de Comercio}

\section{y Negocios}

- Contabilidad

- Sistemas de Gerencia de Información

- Logistica de Transporte

\section{Facultad Ciencias}

- Química

- Clencia de la Atmóstera

- Clencias de la Computación

- Matemáticas

- Microbiología/ Biotecnologia

- Oceanografía

- Física

- Estadistica

\section{Facultad Artes}

Humanidades

- Estudios Asiáticos

- Estudios Religlosos

del Este Medio

- Inglếs

- Artes

- Estudios Franceses,

- Hispánicos,

- Italianos

- Estudios Germánicos

- Historia

- Música

- Filosofía

\section{Facultad de Forestales}

Ciencias Sociales

- Procesamiento

de la Madera
- Antropología y

- Sociología

- Economía

- Geografía

- Lingüística

- Ciencia Política

- Psicología

Fuente: Tripticos de la University of Victoria. Elaboración propia. 
ampliar el ámbito de la formación académica de los futuros profesionales, el acceso al contacto con las últimas tecnologías incorporadas a los procesos productivos, tanto por los estudiantes, como por el personal docente. Las empresas necesitarán la seguridad de contar con trabajadores altamente calificados, y de oportunidades para que éstos continúen aprendiendo. En este contexto las empresas y las universidades deben iniciar un proceso de aproximación que les permita acceder y disponer de una real imagen de las posibilidades y oportunidades que tienen para compartir y obtener beneficios mutuos. En ese sentido ambas organizaciones deben plantearse una revisión de sus valores tradicionales y de los esquemas de funcionamiento, los cuales han incidido para que las universidades y las empresas se hayan mantenido distanciadas por percepciones distorsionadas.

\section{Bíbliografía citada}

Bruce, Evans (1999). Developing a Learning Work. Citado por Cherly Cates y $\mathrm{Pa}$ tricia Jones en The Educational Value of Cooperative Education. The Cooperative Education Inc. Columbia, Maryland, USA. Pág. 37.

CAFCE (1999). Co-operative Education: Helping to do Business in Japan. CAFCE News. Toronto, Canada. Pág. 12-18.

Canadian Association for Co-Operative Education (1996). Accreditation Guide And Application. Toronto, Canada.

Cates Cheryl y Jones, Patricia (1999), Learning Outcomes. The Educational Value of Cooperative Education. The Cooperative Education Associa- tion, Inc. Columbia, Maryland, USA. Pp. 148.

Fortier, Paul (1982). New Approaches - CoOp Pianned by the Employer. Canadian Asosociation for Co-operative Education. 25 Annual Conference. Victoria, Canada. Pág. 111-117.

Marini, Robert (1999), Co-op Enginneering Conections. The newsletter of UBC's Enginneringo Co-op Program. Volumen 1 número 2, mayo 1999, Vancouver, Canada.

Murray, Gillin (1993). The Development of Different Models - A Review. $8^{\text {th }}$ World Conference on Co-Operative Education. Conference Proceeding. Dublin Ireland. pg. 45-47.

Reeve, Rick (1999). A Guide for Developing International Co-op Programs. World Association for Cooperative Education, Inc. Victoria British Columbia, Canada.

University of Alberta, Faculty of Business (1991). Facts E Figures. Brochure. Edmonton, Canada.

University of Alberta, Engineering Co-op (1999). Co-op Employer Handbook. Edmonton, Canada.

University of Alberta - Faculty of Business (1998). Co-op Programs Statistics of Placement Service. Edmonton, Canada.

University of British Columbia (1999). Student benefits from International Experience. Connections the newsletter of UBC's Engineering Co-op Program. Volumen 1 número 3 , septiembre 1999, Vancouver, Canadá.

University of British Columbia - Faculty of Forestry (1999). Wood Products Processing Co-op Program. Employer Information brochure. Vancouver, $\mathrm{Ca}$ nada.

University of Calgary - Career Services (1999). Co-operative Education and 
Cooperación Educativa: relación entre universidad y sector productivo

Espinoza Rodríguez, Rafael L.

Internship Program Activities and Policy Guidelines Information for Faculty and Staff. Calgary, Canada.

University of Calgary - Faculty of Management (1999). Faculty of Management Coop Program Student Handbook 1999 - 2000. Calgary, Canada.

University of Calgary (1999). Management Co-op Program News-letter. Calgary, Canada.

University of Victoria (1996). University of Victoria Co-op Education 20 years old.
UVIC Co-operative Education Programs News Paper. Victoria. Canada.

University of Victoria (1999). Engineering Co-op Education Program Employer Handbook. Victoria, Canada.

Whitmore, June (1996). Geography Co-op Secures $\$ 100,000$ for International Research Project. UVIC Co-operative Education Programs $20^{\text {th }}$ Anniversary. University of Victoria. Victoria, Canadá. 